\title{
Effects of cuing in an "irrelevant" context
}

\author{
ROBERT R. MOWRER and WILLIAM C. GORDON \\ University of New Mexico, Albuquerque, New Mexico
}

\begin{abstract}
Normally, retention of an avoidance response by a rat is impaired when the test context is novel or does not correspond to the training context. Experiment 1 demonstrates that such an impairment of test performance can be alleviated if a rat receives a cuing treatment or reminder of training in the novel test context prior to testing. Experiment 2 indicates that when rats receive avoidance training in one context and then receive a reminder of training in a novel context, they perform more poorly when tested in the training context than do animals that receive no reminder. This finding is discussed in relation to current theories of contextual influence over retention performance.
\end{abstract}

It is clear that contextual stimuli exert strong control over the retention performance of animals. In general, when an animal learns to perform a response in a given context, retention performance is best when subsequent testing occurs in that same context. Deficits in test performance usually result when training and test contexts differ (see Spear, 1978).

Efforts to explain the effects of context on retention range from notions that contextual stimuli function as conditioned or discriminative stimuli (e.g., Rescorla \& Wagner, 1972) to the idea that contextual stimuli serve as retrieval cues for the memory of training experience (e.g., Medin, 1975; Spear, 1973). Regardless of the specific hypothesis advanced, however, virtually all explanations of contextual effects share a common assumption. It is generally assumed that in order for contextual stimuli to gain control over retention performance, these stimuli must be present at the time a response is acquired.

Although such an assumption seems reasonable, recent evidence from our laboratory suggests that it may not always be valid (see Gordon, 1983). These results indicate that contextual stimuli not available at the time of learning, but present when an animal receives a cuing treatment or "reminder" of prior learning, can affect subsequent retention performance substantially. In these studies, the cuing treatments or "reminders" involved confronting animals with a subset of the stimuli that had been present at the time of learning, without exposing the animals to a complete relearning trial. The rationale for such treatments was to provide enough cues to induce retrieval of the training memory without allowing an

This research was supported by a grant from the National Science Foundation to the second author. A portion of this work was presented at the Animal Cognition and Behavior Conference, Norman, Oklahoma, 1982. Reprint requests should be sent to William C. Gordon, Department of Psychology, University of New Mexico, Albuquerque, New Mexico 87131. animal the opportunity for additional learning of the response.

In one such set of experiments (Gordon, McCracken, Dess-Beech, \& Mowrer, 1981), animals were trained to perform an avoidance response in one context and then were tested for retention in either the same or a different context. As in prior studies, animals tested in a novel context performed less well than animals tested in the training context. However, animals given a cuing treatment in the novel test context prior to being tested there performed just as well as animals trained and tested in the same context. Neither cuing in an inappropriate context nor simply exposing animals to the novel test context prior to testing had this facilitatory effect on retention performance. In effect, when animals were cued in a novel context, that context began to function as if it had been the training context. This general finding now has been demonstrated in a variety of situations (e.g., Gordon, 1983; Wittrup \& Gordon, 1982; Gordon, Mowrer, McGinnis, \& McDermott, Note 1).

Our own interpretation of these findings (see Gordon, 1981) follows closely the view of contextual influences proposed by Spear $(1973,1978)$. According to this view, contextual stimuli noticed at the time of training become represented as part of the training memory. In effect, each memory is conceived of as a multidimensional, compound representation. Retrieval of a training memory depends on an animal's confronting some proportion of stimuli that are represented in the training memory. The greater the match between retention test stimuli and stimuli represented in a given memory, the greater is the probability that the memory will be retrieved.

To interpret our findings within this framework, we suggested that not only stimuli noticed at the time of learning, but also stimuli noticed at the time of cuing might become represented in the training memory. Thus, subsequent exposure to the cuing context would be expected to facilitate retrieval by providing 
a match between retention test stimuli and stimuli represented in the training memory. This interpretation is consistent with the finding that a novel cuing context tends to influence retention performance in much the same way as a training context.

This hypothesis, that cuing can result in an addition of contextual representations to a memory, raises questions concerning the context of a training memory following cuing. One possibility is that new contextual representations added at the time of cuing might displace the original representations of the training context. A second possibility is that the addition of new contextual representations simply expands the number of such representations associated with a training memory.

An implication of both of these possibilities is that, following cuing in a novel context, subsequent performance in the training context might be poorer than if no cuing had occurred. In the former case, poor performance would be expected because the context present at the time of testing would fail to match stimuli still represented in the training memory. In the latter case, the process of matching test stimuli to contextual representations might be more difficult or might take longer due to the increased number of representations in the memory. Increased matching time would be expected to impair performance, especially in tasks that require short-latency responses, such as avoidance learning. The purpose of the present studies was to test this notion that cuing can result in impaired performance under some conditions. Specifically, the question addressed was whether cuing in a novel context decreases the capacity of the training context to control retention performance.

\section{EXPERIMENT 1}

The purpose of Experiment 1 was to replicate the basic findings of Gordon et al. (1981) cited above. Specifically, this study attempted to demonstrate that context change between training and testing results in a performance deficit and that this deficit can be reduced by cuing animals in the novel test context prior to testing. Such a replication was necessary because the subject characteristics and parameters employed in Experiments 1 and 2 differed from those used in the previous studies.

\section{Method}

Subjects. The subjects were 48 female albino rats, weighing 240-300 g, obtained from Simonsen Laboratories. The rats were maintained in a vivarium under a 12-h-on/12-h-off light cycle. All treatments occurred during the light portion of this cycle.

Apparatus. The apparatus consisted of two identically constructed active avoidance boxes. Each apparatus contained one white, translucent chamber $(27 \times 13 \times 13 \mathrm{~cm})$ and an identically constructed black chamber. The two chambers were separated by a door that could be lowered (opened), leaving a $3-\mathrm{cm}$ hurdle between the chambers. A running-time meter and a $2-\mathrm{Hz}$ flashing white light $(7.5 \mathrm{~W})$ located directly behind the white chamber could be activated by lowering the chamber door and deactivated by breaking a photobeam located $13 \mathrm{~cm}$ into the black chamber. Each apparatus contained a grid floor made up of .6-cm-diam steel rods set $1.7 \mathrm{~cm}$ apart. A scrambled footshock $(.6 \mathrm{~mA})$ provided by a Grason-Stadler shock generator (Model E1064 GS) could be delivered to the grid floor in the white chamber of either apparatus. Three rooms (A, B, and $\mathrm{C}$ ) were used in the experiment. These rooms differed in terms of size, lighting, odor, ambient noise level, and holding cage (see Table 1). One avoidance apparatus was located in Room A; the other was in Room $B$. In addition, a white, translucent box $(27 \times 13 \times 13 \mathrm{~cm})$, identical to the white chamber of the avoidance apparatus, was used as a "cuing chamber."

Procedure. All rats received active avoidance training followed $48 \mathrm{~h}$ later by a retention test. Avoidance training consisted of placing each rat into the white chamber of the avoidance apparatus with the rat facing the door. After $3 \mathrm{sec}$, the door was lowered, activating the flashing light. If the animal failed to enter the black compartment and break the photobeam within $5 \mathrm{sec}$ after the door was lowered, it received a footshock that lasted until an escape response occurred. An avoidance response consisted of the rat's crossing into the black chamber within $5 \mathrm{sec}$ after the door was lowered. Immediately after either an escape or avoidance response, the door was closed and, after $3 \mathrm{sec}$, the animal was removed to a holding cage for a 30-sec intertrial interval (ITI). The criterion for active avoidance acquisition was five consecutive avoidance responses within 26 training trials. The rats that failed to meet this criterion or failed to receive a footshock within the first 4 training trials were discarded.

Retention testing consisted of five trials, identical to the training trials, except that no shocks were administered regardless of a rat's latency to cross into the black chamber. On each trial, the latency to cross into the black chamber and break the photobeam was recorded. If an animal failed to respond within $60 \mathrm{sec}$ after the door was lowered, a latency of $60 \mathrm{sec}$ was recorded and the trial was terminated. After either crossing into the black chamber or remaining in the white chamber for $60 \mathrm{sec}$, each rat spent a 30sec ITI in a holding cage.

The rats were assigned randomly to one of four treatment groups $(n=12)$. The first group (Group $A-A)$ received active avoidance training and retention testing in Room $A$. The second group (Group B-A) received avoidance training in Room B and testing in Room A. A third group (Group B-A-A) received treatment identical to that of Group B-A, except for receiving a cuing treatment in Room A prior to being tested there. Cuing consisted of

Table 1

Characteristics of Treatment Rooms

\begin{tabular}{|c|c|c|c|}
\hline Characteristics & Room A & Room B & Room C \\
\hline
\end{tabular}


bringing the rat into the testing room (Room A) approximately $4 \mathrm{~min}$ prior to the test. The animal was then placed in the cuing chamber for $15 \mathrm{sec}$, where it was confronted by cues similar to those experienced during training. Following cuing, the rat was removed to a holding cage in Room A for $3.5 \mathrm{~min}$, after which retention testing took place. A final group (Group B-X-A) also was trained in Room B and tested in Room A. However, instead of receiving a cuing treatment in Room A prior to being tested, these animals were confined to a holding cage in Room $\mathbf{A}$ for $3.5 \mathrm{~min}$. Following this confinement, these rats received the cuing treatment in Room C, and then were immediately tested in Room A.

\section{Results and Discussion}

No animal was discarded for failure to meet the training criteria. The mean number of trials to reach the acquisition criterion for each of the treatment conditions was 11.3 for Group A-A, 10.4 for Group B-A, 10.3 for Group B-A-A, and $\mathbf{1 0 . 7}$ for Group B$X-A$. An analysis of variance performed on these data revealed no differences among groups $[F(3,44)$ $=.19$ ]. This indicated that the treatment groups were approximately equivalent in terms of the number of trials needed to acquire the avoidance response. Figure 1 presents the mean $\log$ latency for an animal to cross into the black chamber on the first test trial for each treatment group. An analysis of variance performed on these data indicated a significant effect of treatment condition $[F(3,44)=9.57]$ (in this and all subsequent analyses, a rejection region of .05 was used). A Duncan multiple range test (Duncan, 1955) was used to assess differences among individual treat-

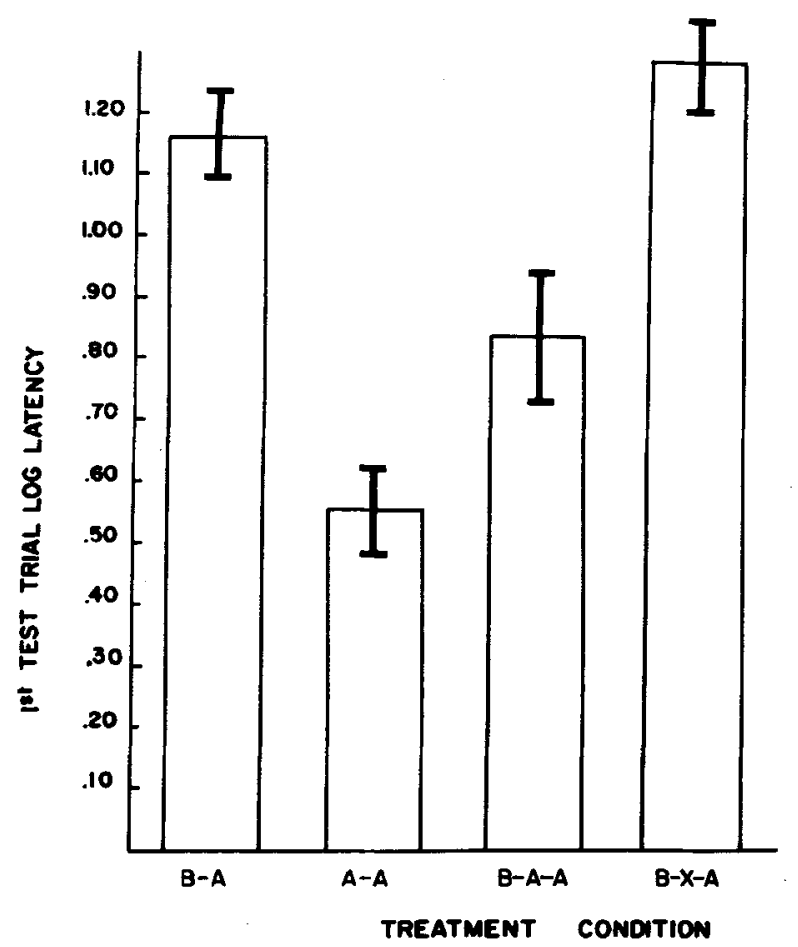

Figure 1. Mean log latencies (in seconds) on first test trial for each group. ment groups. This test revealed that rats in Groups A-A and B-A-A had significantly shorter latencies than did the animals in Groups B-A and B-X-A. There was no difference found between the Group $A-A$ and Group B-A-A animals or between the Group B-A and Group B-X-A animals.

A second measure of retention was each rat's mean $\log$ latency for the five test trials. An analysis of variance performed on these data again indicated a significant effect of treatment condition $[F(3,44)=$ 7.27]. Individual group comparisons revealed the exact pattern of findings that had been found with the first trial $\log$ latencies.

The results of this experiment replicate basic features of the Gordon et al. (1981) studies. Specifically, we again found that changing contexts between training and testing results in a significant performance deficit (cf. Groups B-A and A-A). Furthermore, we showed that this deficit may be reduced by presenting the animal with a cuing treatment in the test context prior to retention testing (cf. Groups B-A and B-A-A). That the reduction of the retention deficit was due to cuing and confinement in the novel test context may be inferred from the comparison of Groups B-A-A and B-X-A. Although animals in Groups B-A-A and B-X-A received equivalent confinement in the test context prior to testing, the cuing treatment occurred in an irrelevant context (Room C) for Group B-X-A. These animals exhibited poor retention performance relative to those cued and confined in the test context (Group B-A-A). These data suggest again that when an animal is given a cuing treatment in a novel context, that context begins to function as if it were the training context.

\section{EXPERIMENT 2}

The first study indicated that contextual stimuli not present at the time of learning but present at the time of cuing can exert control over subsequent retention performance. The second experiment addressed the following question: Once the cuing context acquires control over retention performance, is the capacity of the learning context to affect performance diminished?

\section{Method}

Subjects. The subjects were 60 rats with characteristics identical to those used in the previous experiment.

Apparntus. The apparatus and treatment rooms were the same as those used in the previous experiment.

Procedare. All rats received active avoidance training followed $48 \mathrm{~h}$ later by a retention test. Both training and testing procedures were identical to those used in Experiment 1. In addition to these procedures, all animals were handled prior to training. On each of 3 days prior to training, the rats were removed from the vivarium and carried into a neutral context for approximately $3 \mathrm{~min}$. During this period, each animal was picked up, held for $5 \mathrm{sec}$, replaced in its home cage for $5 \mathrm{sec}$, and again picked up. This was repeated 
six times each day. After completion of each session, the animals were returned to the vivarium. This handling procedure was used to decrease the probability that handling would be uniquely associated with acquisition and would, itself, later serve as an effective cue.

Rats were assigned randomly to one of six treatment conditions $(n=10)$. Groups $\mathbf{A}-\mathbf{A}, \mathbf{B}-\mathbf{A}$, and B-A-A received treatments identical to those used for the corresponding groups in Experiment 1. A fourth group (Group A-B-A) received training in Room A, cuing treatment in Room B, and testing in Room A. Cuing for this group consisted of bringing the animal into the irrelevant cuing context (Group B) approximately $4 \mathrm{~min}$ prior to retention testing in Room A. The rats were placed in the white cuing chamber for $15 \mathrm{sec}$ and then were confined to a holding cage in Room B for $3.5 \mathrm{~min}$. After confinement, the rats were removed to Room $A$ for retention testing. The fifth group (Group A conf B) received training in Room $A$, confinement in Room $B$, and testing in Room A. This group was similar to Group A-B-A, except that the animals were never exposed to the cuing chamber in Room $B$. Instead, these rats were brought into Room B approximately $4 \mathrm{~min}$ prior to being tested and were confined to a holding cage in that room for $3.5 \mathrm{~min}$. These animals then were tested in Room $A$. The final group (Group A conf A) was treated the same as Group A conf $B$, except that confinement occurred in Room $A$ rather than Room B. This group was included to investigate the effects of confinement per se on retention performance.

\section{Results and Discussion}

One rat was discarded and replaced for failure to attain the training criterion. Three rats were discarded and replaced for failure to receive a footshock within the first four training trials. The mean number of trials to reach the training criterion for each group was as follows: Group A-A, 8.1; Group B-A, 8.8; Group B-A-A, 8.9; Group A-B-A, 9.7; Group A conf $B, 10.0$; Group $A$ conf $A, 10.2$. An analysis of variance performed on these data revealed that there were no differences among groups in terms of acquiring the active avoidance response. Figure 2 represents the mean log latency for an animal to cross into the black chamber on Test Trial 1. An analysis of variance performed on these data revealed a significant effect of treatment condition $[F(5,54)=2.77]$. Individual group comparisons (Duncan, 1955) indicated that rats in Groups A-B-A and B-A performed significantly more poorly (i.e., took significantly longer to respond) than did rats in all other groups. An identical pattern of results emerged from the mean log latenzy scores computed for all five test trials. An analysis of variance performed on these data revealed a significant effect of treatment condition $[F(5,54)$ =7.86]. Once again, individual group comparisons indicated that Groups A-B-A and B-A performed significantly more poorly than did all other groups but did not differ from each other.

These data indicate that rats given a cuing treatment in an irrelevant context (Group A-B-A) performed more poorly on a retention test than did animals given no cue (Group A-A). They also performed less well than did rats trained and tested in different contexts but cued in the text context prior to testing (Group B-A-A). That this effect is due to cuing in

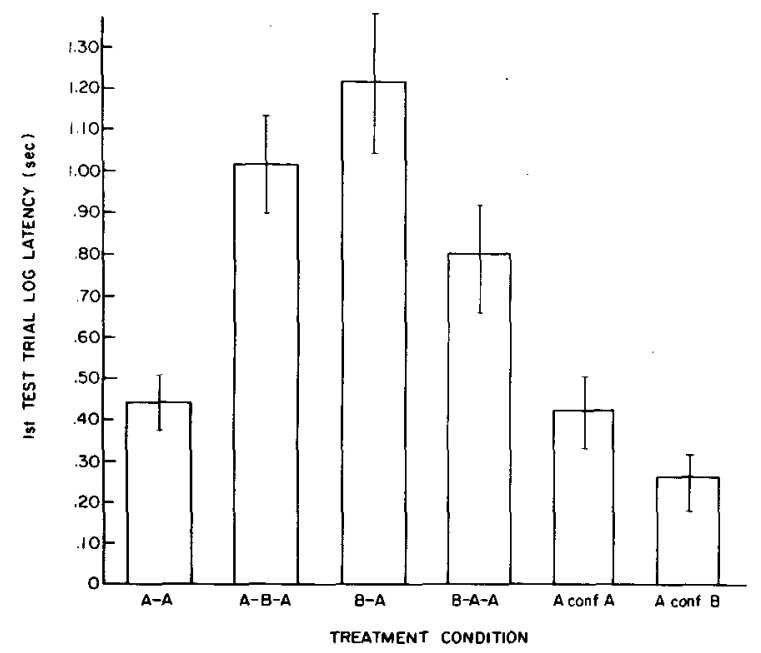

Figure 2. Mean log latencies (in seconds) on first test trial for each group.

the irrelevant context is supported by the relatively good test performance of the rats confined in the irrelevant context without having experienced cuing treatment (Group A conf B). Furthermore, confinement alone produced no detrimental effects on retention performance (cf. Group A conf A).

\section{GENERAL DISCUSSION}

The results of Experiment 1 are consistent with previous findings in showing that a cuing context functions as if it had been the training context (see Gordon et al., 1981, Gordon, 1983, and Wittrup \& Gordon, 1982). In the present case, once animals had been cued in a novel context, subsequent retention performance in that context was almost equivalent to that of animals trained and tested in the same room. As mentioned earlier, this effect can be interpreted within the contextual-cue hypothesis proposed by Spear $(1973,1981)$, if we append to this hypothesis a single assumption.

For example, Spear (1973) suggested that contextual cues noticed at the time of learning become represented as attributes of a training memory. Subsequent exposures to the same cues activate these memory attributes and result in activation or retrieval of the training memory. To explain the present findings within this framework, one need only to assume that stimuli noticed at the time of cuing also become represented as attributes of the training memory. Support for such an assumption is good. First, several studies with animals have shown that training memories are malleable, not only at the time of learning, but also whenever a memory is cued or retrieved (e.g., Gordon, 1981; Lewis, 1979). Second, Loftus and her colleagues (e.g., Loftus, 1979; Loftus \& Zanni, 
1975) have demonstrated that human subjects tend to incorporate new information into previously stored memories if the new information is made available at the time a memory is being retrieved. Thus, it is possible to explain the results of Experiment 1 by assuming that, at the time of cuing, the contextual stimuli present become represented in the memory being cued.

Similarly, it is possible to derive an explanation for these results from at least one other view of contextual stimulus control. For example, according to the Rescorla-Wagner conditioning model (Rescorla \& Wagner, 1972), contextual stimuli combine with the nominal conditioned stimulus (CS) in a learning situation to form a compound CS. These contextual stimuli then compete with the nominal CS for the acquisition of associative strength. In this view, contextual stimuli come to control retention performance because these stimuli acquire, at the time of learning, some capacity to elicit a conditioned response (CR). In the present case, one could assume that the cuing context also attained the capacity for eliciting a CR through a second-order conditioning mechanism.

It is possible, for instance, to assume that a cuing treatment functions as a first-order CS and, thus, elicits a CR when it is presented to an animal. If the cuing presentation occurs in a novel context, the new contextual stimuli may function as second-order CSs and may themselves become capable of eliciting a $\mathrm{CR}$. According to this interpretation, cuing in a novel context facilitates subsequent performance in that context, because the novel contextual stimuli become capable of eliciting CRs appropriate to test performance.

Although such an interpretation is technically viable, this type of explanation encounters a variety of problems. First, direct attempts to show that the cuing context acquires the capacity to elicit fear have not been successful (see Gordon, McCracken, DessBeech, \& Mowrer, 1981). Admittedly, the detection of low levels of fear elicited by contextual stimuli is difficult (e.g., Bouton \& Bolles, 1979). However, if a cuing context acquires only a minimal capacity for eliciting a fear CR, this does raise questions about the importance of such a mechanism for facilitating avoidance test performance.

Another problem with the second-order conditioning explanation is that the present cuing effect appeared to be strongest under those conditions that should have minimized second-order conditioning. We have found that the cuing effect is strong when animals are given a cue in the novel context and then are confined in that context following cuing. We have found in pilot experiments that a failure to confine in the novel context after cuing tends to diminish the cuing effect. Thus, the effective cuing procedure is analogous to exposing animals to a first- and a second-order CS simultaneously and then further exposing the animals to the second-order CS (the novel context) after the offset of the first-order CS (the cuing treatment). In effect, the cuing procedure used in these studies is most analogous to a backward, second-order conditioning paradigm. Attempts to produce a cuing effect by first exposing animals to the novel context and then to the cuing treatment in another context (a forward, trace conditioning paradigm) have been unsuccessful (see Gordon, McCracken, Dess-Beech, \& Mowrer, 1981).

Probably the major problem we have found with the second-order-conditioning explanation is the inability of such an interpretation to deal with the results of Experiment 2, in which cuing in an irrelevant context (i.e., neither the training nor the testing context) actually diminished the control of test performance normally exerted by the training context. If, in Experiments 1 and 2, the cuing context attained control simply by functioning as a second-order CS, then why did the original training context in Experiment 2 decrease its capacity to control performance? The only explanation we can derive from the conditioning hypothesis is that cuing somehow extinguishes associations previously formed to the training context. This argument would be based on the fact that during cuing, one part of the training context (the white chamber) is presented without an accompanying unconditioned stimulus. Thus, the total training context, which consists of room cues and the white chamber, would lose some capacity for eliciting a CR.

This argument suffers, however, from two major weaknesses. First, since the white chamber constitutes only one portion of the training context, one would expect the total context to retain appreciable capacity for eliciting a CR. Yet, performance in the training context is severely interrupted by cuing in a novel context. Second, this explanation predicts that animals trained, cued, and tested in a single context should perform more poorly than animals trained and tested in that context without cuing. This prediction has been shown many times to be incorrect (e.g., Gordon \& Mowrer, 1980; Gordon, Smith, \& Katz, 1979; Wittrup \& Gordon, 1982). If anything, brief exposures to the start chamber of an avoidance apparatus during a retention interval increase rather than decrease subsequent performance. In sum, the conditioning hypothesis appears incapable of explaining the results of Experiment 2 in a convincing manner.

In contrast, these results may well be viewed as being compatible with a retrieval-cue hypothesis (e.g., Spear, 1973), if one assumes that cuing results in the addition of contextual representations to a training memory. As suggested earlier, one might assume that the addition of new representations to a memory displaces some proportion of the original training representations. Thus, as new contextual 
representations are added to a memory, older contextual representations may be lost. If such is the case, contextual stimuli present on a test would fail to match stimuli represented in the training memory and performance would be expected to suffer.

An alternative possibility is that the addition of contextual representations to a memory simply expands the number of attributes associated with that memory. Such an expansion might also be expected to diminish control of performance by the training context. For example, speed of retrieval may depend on how quickly an animal can match environmental stimuli with particular memory attributes. A larger pool of attributes might be expected to slow this matching process and thus to increase retention-test response latencies.

Obviously, these interpretations are only speculations at the present time. Although the present findings are not incompatible with retrieval-cue interpretations of contextual effects, this is largely because such interpretations have been silent concerning the specific effects of cuing on previously established memories. If such models are to give a complete account of contextual influences, they must become more explicit with regard to how memories change as a function of such treatments as cuing.

One additional point should be made. In Experiment 2, each animal was handled extensively prior to training. This procedure was used to eliminate the possibility that handling per se would be associated uniquely with the training experience. In an extensive pilot study run prior to Experiment 2, the conditions of Experiment 2 were employed but without the animals' receiving prior handling. The results were virtually identical to those of Experiment 2, but with one exception. The animals that were simply carried into a novel context but not placed in the white cuing chamber experienced as much of a test deficit as the animals that were cued in the novel context. Apparently, when animals are not handled prior to training, handling itself functions as a cue for the training experience and can duplicate the effects of the nominal cuing treatment. As Experiment 2 demonstrates, prior handling eliminates the cuing effect of handling during a retention interval.

In the final analysis, the present findings indicate that contextual cues not present at the time of learning can affect retention performance. Furthermore, when novel contextual stimuli acquire control over responding through cuing, the control exerted by the training context can be diminished. This is the first report of which we are aware that suggests that cuing can actually debilitate retention performance under selected circumstances. In general, such findings indicate a need for either revision or further elaboration of current theories of contextual influence.

\section{REFERENCE NOTE}

1. Gordon, W. C., Mowrer, R. R., McGinnis, C. P., \& McDermott, M. J. Cue induced proactive interference. Manuscript in preparation, 1983.

\section{REFERENCES}

Bouton, M. E., \& Bolles, R. C. Role of conditioned contextual stimuli in reinstatement of extinguished fear. Journal of Experimental Psychology: Animal Behavior Processes, 1979, 5, 368-378.

Duncan, D. B. Multiple range and multiple F tests. Biometrics, $1955,11,1-42$.

Gordon, W. C. Mechanisms of cue-induced retention enhancement. In N. E. Spear \& R. R. Miller (Eds.), Information processing in animals: Memory mechanisms. Hillsdale, N.J: Erlbaum, 1981.

Gordon, W. C. Malleability of memory in animals. In R. Mellgren (Ed.), Animal cognition and behavior. Amsterdam: NorthHolland, 1983.

Gordon, W. C., McCracken, K. M., Dess-Beech, N., \& Mowrer, R. R. Mechanisms for the cueing phenomenon: The addition of the cueing context to the training memory. Learning and Motivation, 1981, 12, 196-211.

Gondon, W. C., \& Mowner, R. R. The use of an extinction trial as a reminder treatment following ECS. Animal Learning \& Behavior, 1980, 8, 363-367.

Gordon, W. C., Smith, G. J., \& Katz, D. S. Dual effects of response blocking following avoidance learning. Behavior $R e$ search and Therapy, 1979, 17, 479-487.

Lewis, D. J. Psychobiology of active and inactive memory. Psychological Bulletin, 1979, 86, 1054-1083.

LofTus, E. F. The malleability of human memory. American Scientist, 1979, 67, 312-320.

Lortus, E. F., \& ZanNI, G. Eyewitness testimony: The influence of the wording of a question. Bulletin of the Psychonomic Society, 1975, 5, 86-88.

Medin, D. L. A theory of context in discrimination learning. In G. H. Bower (Ed.), The psychology of learning and motivation (Vol. 9). New York: Academic Press, 1975.

Rescorla, R. A., \& Wagner, A. R. A theory of Pavlovian conditioning: Variations in the effectiveness of reinforcement and nonreinforcement. In A. H. Black \& W. F. Prokasy (Eds.), Classical conditioning II: Current research and theory. New York: Appleton-Century-Crofts, 1972.

SPEAR, N. E. Retrieval of memory in animals. Psychological Review, 1973, 80, 163-194.

SPEAR, N. E. The processing of memories: Forgetting and retention. Hillsdale, N.J: Erlbaum, 1978.

Spear, N. E. Extending the domain of memory retrieval. In N. E. Spear \& R. R. Miller (Eds.), Information processing in animals: Memory mechanisms. Hillsdale, N.J: Erlbaum, 1981.

WitTrup, M., \& Gordon, W. C. The alteration of a training memory through cueing. American Journal of Psychology, 1982, 95, 495-507.

(Manuscript received December 6, 1982; revision accepted for publication June 7,1983 .) 